Functiones et Approximatio

55.1 (2016), 7-21

doi: $10.7169 / \mathrm{facm} / 2016.55 .1 .1$

\title{
CHARACTERIZATION OF SPORADIC PERFECT POLYNOMIALS OVER $\mathbb{F}_{2}$
}

\author{
Luis H. Gallardo, Olivier Rahavandrainy
}

\begin{abstract}
We complete, in this paper, the characterization of all known even perfect polynomials over the prime field $\mathbb{F}_{2}$. In particular, we prove that the last two of the eleven known "sporadic" perfect polynomials over $\mathbb{F}_{2}$ are the unique of them of the form $x^{a}(x+1)^{b} M^{2 h} \sigma\left(M^{2 h}\right)$, where $M$ is a Mersenne prime and $a, b, h \in \mathbb{N}^{*}$.
\end{abstract}

Keywords: sum of divisors, polynomials, finite fields, characteristic 2 .

\section{Introduction}

Let $A \in \mathbb{F}_{2}[x]$ be a nonzero polynomial. We say that $A$ is even if it has a linear factor and it is odd otherwise. We define a Mersenne polynomial over $\mathbb{F}_{2}$ as a polynomial of the form $1+x^{a}(x+1)^{b}$, for some positive integers $a, b$. If such a polynomial is irreducible, we say that it is a Mersenne prime.

Let $\omega(A)$ denote the number of distinct irreducible (or prime) factors of $A$ over $\mathbb{F}_{2}$ and let $\sigma(A)$ denote the sum of all divisors of $A$ ( $\sigma$ is a multiplicative function). If $\sigma(A)=A$, then we say that $A$ is a perfect polynomial. The notion of perfect polynomials is introduced ([3]) by E.F. Canaday in 1941 and extended by J.T.B. Beard Jr. et al. in several directions ([1], [2]). We are interested in this subject since a few years and have obtained some results ([4], [5], [6], [7], [8]).

If $A \in \mathbb{F}_{2}[x]$ is nonconstant and perfect, then $\omega(A) \geqslant 2$ (Lemma 2.1). Moreover ([3]), the only perfect polynomials $A$ over $\mathbb{F}_{2}$ with $\omega(A)=2$ are those of the form $\left(x^{2}+x\right)^{2^{n}-1}$, for some positive integer $n$. We call them "trivial" perfect. Contrary to the integer case in which any even perfect number has exactly two distinct prime factors, we do not know the value of $\omega(A)$ for a non-trivial even perfect polynomial $A \in \mathbb{F}_{2}[x]$. We are unable to describe a general form of such polynomials in terms of Mersenne primes. However, as discussed below, with only two exceptions, all known non-trivial even perfect polynomials have factorizations with Mersenne primes as odd divisors.

2010 Mathematics Subject Classification: primary: 11T55; secondary: 11T06 
In the rest of the paper:

(a) For $S \in \mathbb{F}_{2}[x]$, we denote by $\bar{S}$ the polynomial obtained from $S$ with $x$ replaced by $x+1: \bar{S}(x)=S(x+1)$.

(b) We denote by $\alpha$ a root of the irreducible polynomial $x^{2}+x+1$ in a fixed algebraic closure of $\mathbb{F}_{2}$. In other words: $\mathbb{F}_{4}:=\mathbb{F}_{2}[\alpha]$, where $\mathbb{F}_{4}$ is the finite field with 4 elements.

Remark 1.1. In other words, for any $S \in \mathbb{F}_{2}[x]$, one has

$$
S(\alpha) \neq 0 \Longleftrightarrow \operatorname{gcd}\left(S(x), x^{2}+x+1\right)=1 .
$$

As usual, $\mathbb{N}$ (resp. $\mathbb{N}^{*}$ ) denotes the set of nonnegative integers (resp. of positive integers).

We proved ([5], [6]) that any nonconstant and non-trivial perfect polynomial $A \in \mathbb{F}_{2}[x]$ with $\omega(A) \leqslant 4$ is even and takes one of the following forms:

$$
\begin{gathered}
T_{1}=x^{2}(x+1) M_{1}, \quad T_{2}=\bar{T}_{1}, \quad T_{3}=x^{4}(x+1)^{3} M_{3}, \quad T_{4}=\bar{T}_{3}, \\
C_{1}=x^{2}(x+1)\left(x^{4}+x+1\right) M_{1}^{2}, \quad C_{2}=\bar{C}_{1}, \quad C_{3}=x^{4}(x+1)^{4} M_{3} \overline{M_{3}}=\bar{C}_{3}, \\
C_{4}=x^{6}(x+1)^{3} M_{2} \overline{M_{2}}, \quad C_{5}=\bar{C}_{4},
\end{gathered}
$$

where $M_{j}=1+x(x+1)^{j}, j=1,2,3$.

Moreover, there are only two more known even perfect polynomials with five prime factors: $S_{1}=x^{4}(x+1)^{6} M_{2} \overline{M_{2}} M_{3}$ and $S_{2}=\bar{S}_{1}$.

These eleven polynomials are the only known non-trivial perfect polynomials over $\mathbb{F}_{2}$. We call them "sporadic" perfect.

We immediately remark that, except for $C_{1}$ and $C_{2}$, all of them are of the form $x^{a}(x+1)^{b} P_{1} \cdots P_{r}$ where $a, b \in \mathbb{N}^{*}$ and each $P_{j}$ is a Mersenne prime. These two exceptions $C_{1}, C_{2}$ show that contrary to the case of integers, there exist even perfect polynomials over $\mathbb{F}_{2}$ which are divisible by a non Mersenne prime. We showed ([9], Theorem 1.1) that these nine known polynomials are the unique perfect polynomials that have factorizations involving Mersenne primes as odd prime divisors raised to powers of the form $2^{n}-1$. We want to better understand the factorisation of the last two sporadic perfect polynomials $C_{1}$ and $C_{2}$. We obviously see that $C_{1}=x^{2}(x+1) M_{1}^{2} \sigma\left(M_{1}^{2}\right)$ and $C_{2}=x(x+1)^{2} M_{1}^{2} \sigma\left(M_{1}^{2}\right)$. So, it is natural to think of perfect polynomials of the form $x^{a}(x+1)^{b} M^{2 h} \sigma\left(M^{2 h}\right)$, where $M$ is a Mersenne prime and $a, b, h \in \mathbb{N}^{*}$. Proposition 3.5 implies that, in this case, $M \in\left\{M_{1}, M_{3}\right\}$ and the polynomial $\sigma\left(\sigma\left(M^{2 h}\right)\right)$ must be of the form $x^{u}(x+1)^{v} M^{w}$, for some $u, v, w \in \mathbb{N}^{*}$. Theorem 1.3 shows that $M \neq M_{3}$.

In this paper, we characterize in Theorem 1.4 (with the help of Theorems 1.2 and 1.3) the polynomials $C_{1}$ and $C_{2}$, as the unique perfect polynomials that are of the form $x^{a}(x+1)^{b} M^{2 h} \sigma\left(M^{2 h}\right)$, where $M$ is a Mersenne prime.

Theorem 1.2. If $M=1+x+x^{2}$ and if $\sigma\left(\sigma\left(M^{2 h}\right)\right)=x^{u}(x+1)^{v} M^{w}$, then $u=v$ and $w$ is odd. Moreover, if $u=v=1$, then $w=h=1$.

Theorem 1.3. If $M=1+x+\cdots+x^{4}$, then for any $a, b, h \in \mathbb{N}^{*}$, there exists no perfect polynomial over $\mathbb{F}_{2}$ of the form $x^{a}(x+1)^{b} M^{2 h} \sigma\left(M^{2 h}\right)$. 
Theorem 1.4. Let $A=x^{a}(x+1)^{b} M^{2 h} \sigma\left(M^{2 h}\right)$ be an even polynomial over $\mathbb{F}_{2}$, where $M$ is a Mersenne prime and $h \in \mathbb{N}^{*}$. Then $A$ is perfect if and only if $M=x^{2}+x+1, h=1$ and $(a, b) \in\{(1,2),(2,1)\}$ so that $\{A, \bar{A}\}=\left\{C_{1}, C_{2}\right\}$.

\section{Preliminaries}

Some of the following results are obvious or well known, so we omit their proofs.

Lemma 2.1 ([4, Lemma 2.3]). If $A=P_{1}^{h_{1}} \cdots P_{r}^{h_{r}} Q_{1}^{k_{1}} \cdots Q_{s}^{k_{s}}$ is a nonconstant perfect polynomial over $\mathbb{F}_{2}$ such that:

$$
\left\{\begin{array}{l}
P_{1}, \ldots, P_{r}, Q_{1}, \ldots, Q_{s} \text { are distinct and irreducible, } \\
\operatorname{deg}\left(P_{1}\right)=\cdots=\operatorname{deg}\left(P_{r}\right)<\operatorname{deg}\left(Q_{1}\right) \leqslant \cdots \leqslant \operatorname{deg}\left(Q_{s}\right)
\end{array}\right.
$$

then $r$ is even.

Lemma 2.2. If $A=A_{1} A_{2}$ is perfect over $\mathbb{F}_{2}$ and if $\operatorname{gcd}\left(A_{1}, A_{2}\right)=1$, then $A_{1}$ is perfect if and only if $A_{2}$ is perfect.

Lemma 2.3. If $A$ is perfect over $\mathbb{F}_{2}$, then the polynomial $\bar{A}$ is also perfect over $\mathbb{F}_{2}$.

Lemma 2.4. If $A$ is an odd perfect polynomial over $\mathbb{F}_{2}$, then $A$ is a square.

Lemma 2.5 ([3, Theorem 8]). If any irreducible factor of $1+x+\cdots+x^{2 n}$ is of the form $x^{a}(x+1)^{b}+1$, then $n \in\{1,2,3\}$.

Lemma 2.6. Let $h$ be a positive integer and let $M \in \mathbb{F}_{2}[x]$ be a Mersenne prime. Then, $\sigma\left(x^{2 h}\right)$ and $\sigma\left(M^{2 h}\right)$ are both odd and squarefree.

Proof. The facts: $\sigma\left(x^{2 h}\right)$ and $\sigma\left(M^{2 h}\right)$ are odd and $\sigma\left(x^{2 h}\right)$ is squarefree are immediate. Put $H=\sigma\left(M^{2 h}\right)=M^{2 h}+\cdots+M+1$. By differentiating $H$, one has: $H^{\prime}=M^{\prime} \cdot\left(M^{h-1}+\cdots+M+1\right)^{2}$.

We show that $\operatorname{gcd}\left(H, H^{\prime}\right)=1$. Suppose that $\beta$ is a common root of $H$ and $H^{\prime}$ in a suitable field extension of $\mathbb{F}_{2}$. It is obvious that $M^{\prime}(\beta) \neq 0$ since $M^{\prime}$ has at most two roots: 0,1 and $H(0)=H(1)=1$.

Hence, $\beta$ satisfies: $\left(M^{2 h}+\cdots+M+1\right)(\beta)=0=\left(M^{h-1}+\cdots+M+1\right)(\beta)$. Thus, $0=H(\beta)=\left(M^{2 h}+\left(M^{h}+1\right)\left(M^{h-1}+\cdots+M+1\right)\right)(\beta)=M^{2 h}(\beta)+0$. So $M(\beta)=0$ and $0=H(\beta)=1$, which is impossible.

Corollary 2.7. Let $M \in \mathbb{F}_{2}[x]$ be a Mersenne prime such that $\sigma\left(\sigma\left(M^{2 h}\right)\right)=$ $x^{u}(x+1)^{v} M^{w}$. Then, any irreducible divisor of $\sigma\left(M^{2 h}\right)$ is of the form $1+x^{a_{i}}(x+1)^{b_{i}}$ or $1+x^{c_{i}}(x+1)^{d_{i}} M^{e_{i}}$, for some positive integers $a_{i}, b_{i}, c_{i}, d_{i}, e_{i}$.

Proof. Since $\sigma\left(M^{2 h}\right)$ is odd and squarefree, we get $\sigma\left(M^{2 h}\right)=V_{1} \cdots V_{r}$, where $r \in \mathbb{N}^{*}$ and each $V_{i}$ is odd and irreducible. Hence, $x^{u}(x+1)^{v} M^{w}=\sigma\left(\sigma\left(M^{2 h}\right)\right)=$ $\left(1+V_{1}\right) \cdots\left(1+V_{r}\right)$. Therefore, for any $i, 1+V_{i}$ is of the form $x^{a_{i}}(x+1)^{b_{i}}$ or $x^{c_{i}}(x+1)^{d_{i}} M^{e_{i}}$ for some $a_{i}, b_{i}, c_{i}, d_{i}, e_{i} \in \mathbb{N}$. The irreducibility of $V_{i}$ and the fact that it is odd imply that $a_{i}, b_{i}, c_{i}, d_{i}, e_{i}$ must be positive. 
Lemma 2.8. [[10], Theorem 7)] Let $f \in \mathbb{F}_{2}[x]$ be a squarefree polynomial of degree $n$. Then

i) $f\left(1+x+x^{2}\right)$ is also squarefree.

ii) $\omega\left(f\left(1+x+x^{2}\right)\right)$ is even if and only if $(-1)^{n} F(3,4) \equiv 1 \bmod 8$, where $F(x, y)$ is the homogeneous lift of $f$ to $\mathbb{Z}[x]$.

Corollary 2.9. If $M=x^{2}+x+1$, then for any $h \in \mathbb{N}^{*}$, the number $\omega\left(\sigma\left(M^{2 h}\right)\right)$ of irreducible divisors of $\sigma\left(M^{2 h}\right)$ is odd.

Proof. Since the homogeneous lift of $\sigma\left(x^{2 h}\right)$ to $\mathbb{Z}[x]$ equals

$$
F(x, y)=\frac{x^{2 h+1}-y^{2 h+1}}{x-y},
$$

and $F(3,4) \equiv 5 \not \equiv 1(\bmod 8)$ the assertion follows from Lemma 2.8-ii).

\section{The proof of Theorem 1.4}

We shall now show how our main result, Theorem 1.4, follows from Theorems 1.2 and 1.3. We start with a few technical lemmas.

\subsection{Useful facts}

Lemma 3.1. Let $S \in \mathbb{F}_{2}[x]$ be irreducible such that $S=\bar{S}$ and $S(\alpha) \neq 0$, then $S(\alpha)=1$ and $x^{2}+x+1$ divides $1+S$.

Proof. Observe that from Remark 1.1 one has $\operatorname{gcd}\left(S(x), x^{2}+x+1\right)=1$. Write $S(x)=Q(x)\left(x^{2}+x+1\right)+R(x)$ with $Q(x), R(x) \in \mathbb{F}_{2}[x]$ and $R(x)=a+b x \neq 0$. Thus, $a+b \alpha=S(\alpha)=S(\alpha+1)=a+b(\alpha+1)$. It follows that $b=0$. Therefore $0 \neq S(\alpha)=a \in \mathbb{F}_{2}$. Thus, $a=1$, thereby proving the first assertion. Since $(1+S)(\alpha)=0,1+S(x)$ is divisible by the minimal polynomial of $\alpha$ over $\mathbb{F}_{2}$. In other words, $x^{2}+x+1$ divides $1+S(x)$. This completes the proof of the lemma.

Corollary 3.2. Let $M=1+x+x^{2}, h \in \mathbb{N}^{*}$ and $H=\sigma\left(M^{2 h}\right)$. Then there exists an irreducible divisor $P$ of $H$ such that $P=\bar{P}$ and $P(\alpha)=1$.

Proof. First, $H=\bar{H}$ because $M=\bar{M}$. By Lemma 2.6, $H=P_{1} P_{2} \cdots P_{r}$, where each $P_{j}$ is irreducible. Since $H=\bar{H}$, one has: $P|H \Rightarrow \bar{P}| H$.

If for any $j, P_{j} \neq \overline{P_{j}}$, then we may write without loss of generality:

$$
H=P_{1} \overline{P_{1}} P_{2} \overline{P_{2}} \cdots P_{s} \overline{P_{s}}
$$

and $\omega(H)=2 s$, which contradicts Corollary 2.9. Moreover, any irreducible divisor $P$ of $\sigma(H)$ is distinct from $M$ and thus satisfies: $P(\alpha) \neq 0$. We get our corollary from Lemma 3.1.

Corollary 3.3. For any $h \in \mathbb{N}^{*}, M=1+x+x^{2}$ divides $\sigma\left(\sigma\left(M^{2 h}\right)\right)=\sigma(H)$. 
Proof. By Corollary 3.2, let $P$ be irreducible such that $P \| H$ and $P=\bar{P}$. Then, $1+P$ divides $\sigma(H)$, and from Lemma 3.1, $M$ divides $1+P$.

Lemma 3.4. If $M=x^{2}+x+1$ and if $T \in \mathbb{F}_{2}[x]$ are such that $T=\bar{T}$. Then

i) there exists $S \in \mathbb{F}_{2}[x]$ such that $T=S(M)$.

ii) $\overline{\sigma(T)}=\sigma(T)$.

iii) If $x^{u} \| T$ and $(x+1)^{v} \| T$, then $u=v$.

Proof. i): By induction on the degree of $T$, we can prove that there exists $R \in$ $\mathbb{F}_{2}[x]$ such that $T=R(x(x+1))$. It suffices then to take $S(x)=R(x+1)$.

ii) is immediate.

iii): Put $T=x^{u}(x+1)^{v} U$, where $U$ is an odd polynomial. Since $\bar{T}=T$, one has: $x^{v}(x+1)^{u} \bar{U}=\bar{T}=T=x^{u}(x+1)^{v} U$. We are done.

\subsection{The proof}

Assume, in this section, that the polynomial $A=x^{a}(x+1)^{b} M^{2 h} \sigma\left(M^{2 h}\right)$ is perfect over $\mathbb{F}_{2}$, with $M$ a Mersenne prime, $a, b, h \in \mathbb{N}^{*}$ and $a \leqslant b$. We set $M_{1}=1+x+x^{2}$ and $M_{3}=1+x+\cdots+x^{4}$.

For $r \in \mathbb{N}$, put $U_{2 h}=\sigma\left(\sigma\left(M^{2 h}\right)\right)$ and

$$
\begin{array}{lll}
S_{r, h}=x^{2^{r+1}}(x+1)^{2^{r+1}} M^{2 h-2^{r+1}}, & T_{r, h}=x^{2^{r}}(x+1)^{2^{r}} M^{2 h-2^{r}} & \text { if } M=M_{1}, \\
S_{r, h}=x^{3 \cdot 2^{r}}(x+1)^{2^{r}} M^{2 h-2^{r}}, & T_{r, h}=x^{2^{r}}(x+1)^{3 \cdot 2^{r}} M^{2 h-2^{r}} & \text { if } \quad M=M_{3} .
\end{array}
$$

\section{Proposition 3.5.}

i) $M$ divides at least one of $\sigma\left(x^{a}\right)$ and $\sigma\left((x+1)^{b}\right)$.

ii) One has either $M=M_{1}$ or $M=M_{3}$.

iii) If $M=M_{1}$, then for some $r \in \mathbb{N}$, we have $\left(a=b=3 \cdot 2^{r}-1, U_{2 h}=S_{r, h}\right)$ or $\left(a=2 \cdot 2^{r}-1, b=3 \cdot 2^{r}-1, U_{2 h}=T_{r, h}\right)$.

iv) If $M=M_{3}$ then $U_{2 h} \in\left\{S_{r, h}, T_{r, h}\right\}$, for some $r \in \mathbb{N}$.

Proof. i): Put $A=x^{a}(x+1)^{b} M^{2 h} \sigma\left(M^{2 h}\right), a+1=2^{s} u$ and $b+1=2^{r} v$, with $s, r \geqslant 0, u, v$ odd. One has:

$$
\begin{aligned}
\sigma\left(x^{a}\right) & =1+x+\cdots+x^{a}=(1+x)^{2^{s}-1}\left(1+x+\cdots+x^{u-1}\right)^{2^{s}}, \\
\sigma\left((x+1)^{b}\right) & =x^{2^{r}-1}\left(1+(x+1)+\cdots+(x+1)^{v-1}\right)^{2^{r}} .
\end{aligned}
$$

We remark that the four polynomials $x, x+1, M$ and $\sigma\left(M^{2 h}\right)$ are pairwise coprime. Hence, $\sigma(A)=\sigma\left(x^{a}\right) \sigma\left((x+1)^{b}\right) \sigma\left(M^{2 h}\right) \sigma\left(\sigma\left(M^{2 h}\right)\right)$.

Since $A$ is perfect, we get

$$
x^{a}(x+1)^{b} M^{2 h} \sigma\left(M^{2 h}\right)=\sigma\left(x^{a}\right)_{\sigma}\left((x+1)^{b}\right) \sigma\left(M^{2 h}\right) \sigma\left(\sigma\left(M^{2 h}\right)\right),
$$

so that $x^{a}(x+1)^{b} M^{2 h}=\sigma\left(x^{a}\right) \sigma\left((x+1)^{b}\right) \sigma\left(\sigma\left(M^{2 h}\right)\right)$.

If $M \nmid \sigma\left(x^{a}\right)$ and $M \nmid \sigma\left((x+1)^{b}\right)$, then $M^{2 h}$ divides $\sigma\left(\sigma\left(M^{2 h}\right)\right)$. Thus,

$$
M^{2 h}=\sigma\left(\sigma\left(M^{2 h}\right)\right), \quad M^{2 h} \sigma\left(M^{2 h}\right) \text { is odd and perfect, }
$$

which is impossible by Lemmas 2.4 and 2.6. 
ii): If $M \mid \sigma\left(x^{a}\right)$, then $M=1+x+\cdots+x^{u-1}$. Hence, by Lemma $2.5, u=3$ or $u=5$.

If $M \mid \sigma\left((x+1)^{b}\right)$, then as above: $M \in\left\{M_{1}, M_{3}\right\}$.

iii): From i), $M=M_{1}$ must divide at least one of $\sigma\left(x^{a}\right)$ and $\sigma\left((x+1)^{b}\right)$.

- If $M \mid \sigma\left(x^{a}\right)$ and $M \mid \sigma\left((x+1)^{b}\right)$, then $M=1+x+\cdots+x^{u-1}=1+(x+$ $1)+\cdots+(x+1)^{v-1}$. Hence, $u=v=3$. Thus, $s \leqslant r, 2^{r}-1 \leqslant a=3 \cdot 2^{s}-1$ and $2^{s}-1 \leqslant b=3 \cdot 2^{r}-1$. It follows that $s \leqslant r \leqslant s+1$. We get $U_{2 h}=S_{r, h}$ if $s=r$. If $r=s+1$, then

$$
a=3 \cdot 2^{s}-1, \quad b=6 \cdot 2^{s}-1, \quad \sigma\left(\sigma\left(M^{2 h}\right)\right)=x^{2^{s}} \cdot(x+1)^{5 \cdot 2^{s}} \cdot M^{2 h-3 \cdot 2^{s}},
$$

which is impossible by Lemma 3.4 .

- If $M \mid \sigma\left(x^{a}\right)$ but $M \nmid \sigma\left((x+1)^{b}\right)$, then $u=3, v=1$. Thus, $2^{r}-1 \leqslant a=$ $3 \cdot 2^{s}-1 \leqslant b=2^{r}-1$ and $2^{s}-1 \leqslant b=2^{r}-1$. So $r \leqslant s+1<r$, which is impossible.

- If $M \nmid \sigma\left(x^{a}\right)$ but $M \mid \sigma\left((x+1)^{b}\right)$, then $u=1, v=3$. Thus, $2^{r}-1 \leqslant a=$ $2^{s}-1 \leqslant b=3 \cdot 2^{r}-1$ and $2^{s}-1 \leqslant b=3 \cdot 2^{r}-1$. So $r \leqslant s \leqslant r+1$. If $s=r$, then

$$
a=2^{r}-1, \quad b=3 \cdot 2^{r}-1 \quad \text { and } \quad \sigma\left(\sigma\left(M^{2 h}\right)\right)=(x+1)^{2^{r+1}} \cdot M^{2 h-2^{r}},
$$

which is impossible by Lemma 3.4. We get $U_{2 h}=T_{r, h}$ if $s=r+1$.

iv): Now, we suppose that $M=M_{3}$.

- If $M \mid \sigma\left(x^{a}\right)$ and $M \mid \sigma\left((x+1)^{b}\right)$, then $M=1+x+\cdots+x^{u-1}=1+(x+$ 1) $+\cdots+(x+1)^{v-1}$, which is impossible.

- If $M \mid \sigma\left(x^{a}\right)$ but $M \nmid \sigma\left((x+1)^{b}\right)$, then $u=5, v=1$. Thus, $2^{r}-1 \leqslant a=$ $5 \cdot 2^{s}-1$ and $2^{s}-1 \leqslant b=2^{r}-1$. So $s \leqslant r \leqslant s+2$.

- If $r=s$, then

$$
x^{a}(x+1)^{b} M^{2 h}=\sigma\left(x^{a}\right) \sigma\left((x+1)^{b}\right) U_{2 h}=(x+1)^{2^{s}-1} M^{2^{s}} x^{2^{s}-1} U_{2 h},
$$

so that $U_{2 h}=x^{4 \cdot 2^{s}} M^{2 h-2^{s}}$. Hence, any irreducible divisor of $\sigma\left(M^{2 h}\right)$ is of the form $1+x^{c} M^{d}$, which is impossible by Corollary 2.7 .

- If $r=s+1$, then

$$
a=5 \cdot 2^{s}-1, \quad b=2 \cdot 2^{s}-1, \quad U_{2 h}=x^{3 \cdot 2^{s}}(x+1)^{2^{s}} M^{2 h-2^{s}}=S_{s, h} .
$$

- If $r=s+2$, then

$$
a=5 \cdot 2^{s}-1, \quad b=4 \cdot 2^{s}-1, \quad U_{2 h}=x^{2^{s}}(x+1)^{3 \cdot 2^{s}} M^{2 h-2^{s}}=T_{s, h} .
$$

- If $M \nmid \sigma\left(x^{a}\right)$ but $M \mid \sigma\left((x+1)^{b}\right)$, then $u=1, v=5$. As above, we get $r \leqslant s \leqslant r+2$ and $U_{2 h} \in\left\{S_{s, h}, T_{s, h}\right\}$.

We can now finish the proof of Theorem 1.4. If $A$ is perfect, then the case $M=M_{3}$ is excluded by Theorem 1.3. From Theorem 1.2, we get: $\sigma\left(\sigma\left(M^{2 h}\right)\right)=$ $x^{u}(x+1)^{u} M^{w}$, for some $u, w \in \mathbb{N}^{*}$, with $w$ odd.

Proposition 3.5-iii) gives: $\sigma\left(\sigma\left(M^{2 h}\right)\right)=T_{r, h}=x^{2^{r}} \cdot(x+1)^{2^{r}} \cdot M^{2 h-2^{r}}$, with $r=0$ and $a=2 \cdot 2^{r}-1=1, b=3 \cdot 2^{r}-1=2$. Again, Theorem 1.2 implies that $h=1$ and we get our theorem. 


\section{Proof of Theorem 1.2}

In this section, we take $M=1+x+x^{2}$. Primo, we see that $u=v$ since $\sigma\left(\sigma\left(M^{2 h}\right)\right)=\overline{\sigma\left(\sigma\left(M^{2 h}\right)\right)}$. Secundo, Lemma 4.1 below states that $w=h=1$ if $u=v=1$. It remains then to show that $w$ is odd.

Lemma 4.1. If $\sigma\left(\sigma\left(M^{2 h}\right)\right)=x(x+1) M^{2 h-1}$, then $h=1$.

Proof. We may write, by Corollary 2.7: $\sigma\left(M^{2 h}\right)=V_{1} \cdots V_{r}$, where each $V_{i}$ is odd and irreducible of the form $1+x^{a_{i}}(x+1)^{b_{i}}$ or $1+x^{c_{i}}(x+1)^{d_{i}} M^{e_{i}}$, for some positive integers $r, a_{i}, b_{i}, c_{i}, d_{i}, e_{i}$. If $r \geqslant 2$, then $x^{2}$ divides $\sigma\left(\sigma\left(M^{2 h}\right)\right)$, which is impossible. So, $r=1$ and $\sigma\left(M^{2 h}\right)=V_{1}=1+x(x+1) M^{2 h-1}$. Hence, $M^{2 h}+\cdots+M=$ $\sigma\left(M^{2 h}\right)+1=x(x+1) M^{2 h-1}$ and $2 h-1=1$.

Notation 4.2. For a polynomial $S \in \mathbb{F}_{2}[x]$ of degree $s$, we denote by $\alpha_{k}(S)$ the coefficient of $x^{s-k}$ in $S, 1 \leqslant k \leqslant s$.

Lemma 4.3. Let $S \in \mathbb{F}_{2}[x]$ such that $\operatorname{gcd}\left(S, x(x+1)\left(x^{2}+x+1\right)\right)=1$, then $\alpha_{1}(\sigma(S))=\alpha_{1}(S)$ and $\alpha_{2}(\sigma(S))=\alpha_{2}(S)$.

Proof. In this case, $\sigma(S)=S+T$, where $\operatorname{deg}(T) \leqslant \operatorname{deg}(S)-3$. We are done.

Lemma 4.4. If $u, v, w \in \mathbb{N}^{*}$, then one has modulo 2 :

$$
\begin{gathered}
\alpha_{2}\left(M^{w}\right)=\frac{w(w+1)}{2}, \quad \alpha_{2}\left(\sigma\left(M^{w}\right)\right)=1+\alpha_{2}\left(M^{w}\right), \\
\alpha_{2}\left(x^{u}(x+1)^{v} M^{w}\right)=\frac{v(v-1)}{2}+\frac{w(w+1)}{2}+v w .
\end{gathered}
$$

Proof. $M^{w}=\left(\left(x^{2}+x\right)+1\right)^{w}=\left(x^{2}+x\right)^{w}+w\left(x^{2}+x\right)^{w-1}+\cdots$. So

$$
M^{w}=x^{2 w}+w x^{2 w-1}+\left(\begin{array}{l}
w \\
2
\end{array}\right) x^{2 w-2}+\cdots+w\left(x^{2}+x\right)^{w-1}+\cdots
$$

and

$$
\begin{aligned}
\alpha_{2}\left(M^{w}\right) & =\left(\begin{array}{l}
w \\
2
\end{array}\right)+w=\left(\begin{array}{c}
w+1 \\
2
\end{array}\right), \\
\alpha_{2}\left(\sigma\left(M^{w}\right)\right) & =\alpha_{2}\left(M^{w}+M^{w-1}+\cdots\right)=\alpha_{2}\left(M^{w}\right)+1 .
\end{aligned}
$$

We have $\alpha_{2}\left(x^{u}(x+1)^{v} M^{w}\right)=\alpha_{2}\left((x+1)^{v} M^{w}\right)$ and $(x+1)^{v} M^{w}=\left(x^{v}+v x^{v-1}+\left(\begin{array}{l}v \\ 2\end{array}\right) x^{v-2}+\cdots\right)\left(x^{2 w}+w x^{2 w-1}+\left(\begin{array}{c}w+1 \\ 2\end{array}\right) x^{2 w-2}+\cdots\right)$

Hence

$$
\alpha_{2}\left((x+1)^{v} M^{w}\right)=\frac{v(v-1)}{2}+\frac{w(w+1)}{2}+v w
$$


We can now finish the proof of Theorem 1.2. We suppose that $\sigma\left(\sigma\left(M^{2 h}\right)\right)=$ $x^{u}(x+1)^{u} M^{w}$ where $w=2 \ell$ is even. By comparing degrees, we get: $u=2 d$ is even and $h=\ell+d$. We apply Lemmas 4.3 and 4.4 to $S=\sigma\left(M^{2 h}\right)$ and to $M^{2 h}$. We get modulo 2: $\alpha_{2}\left(\sigma\left(\sigma\left(M^{2 h}\right)\right)\right)=\alpha_{2}\left(\sigma\left(M^{2 h}\right)\right)=1+\alpha_{2}\left(M^{2 h}\right)=1+h$.

On the other hand, still by Lemma 4.4 , we obtain:

$$
\alpha_{2}\left(x^{u}(x+1)^{u} M^{2 d}\right) \equiv \ell+d \bmod 2 .
$$

So, we get the contradiction:

$$
1+h \equiv \alpha_{2}\left(\sigma\left(\sigma\left(M^{2 h}\right)\right)\right)=\alpha_{2}\left(x^{u}(x+1)^{u} M^{2 d}\right) \equiv \ell+d=h \quad \bmod 2 .
$$

\section{Proof of Theorem 1.3}

In this section, we set $M=1+x+x^{2}+x^{3}+x^{4}$ and for $h \in \mathbb{N}^{*}$ and $r \in \mathbb{N}$ :

$U_{2 h}=\sigma\left(\sigma\left(M^{2 h}\right)\right), \quad S_{r, h}=x^{3 \cdot 2^{r}}(x+1)^{2^{r}} M^{2 h-2^{r}}, \quad T_{r, h}=x^{2^{r}}(x+1)^{3 \cdot 2^{r}} M^{2 h-2^{r}}$.

The main idea of the proof is similar (but technically more complicated) as that of Theorem 1.2: Proposition 3.5-iv) implies that $U_{2 h} \in\left\{S_{r, h}, T_{r, h}\right\}$, for some $r \in \mathbb{N}$. If $r \in\{0,1\}$, we shall show directly that this is not possible. For $r \geqslant 2$, we shall see that this is also impossible by proving that $\alpha_{k}\left(U_{2 h}\right) \neq \alpha_{k}\left(S_{r, h}\right)$, $\alpha_{l}\left(U_{2 h}\right) \neq \alpha_{l}\left(T_{r, h}\right)$ for some $1 \leqslant k, l \leqslant 5$ (see Notation 4.2 and Corollaries 5.12 , $5.14,5.16$ and 5.18). The rough (trivial) idea is that two polynomials are equal if and only if they have the same coefficients.

\subsection{Case $r \in\{0,1\}$}

We prove, directly, that if $r \in\{0,1\}$, then $U_{2 h} \neq S_{r, h}, T_{r, h}$, for any $h \in \mathbb{N}^{*}$.

\section{Case $r=0$}

- If $U_{2 h}=S_{0, h}=x^{3}(x+1) M^{2 h-1}$, then $\sigma\left(M^{2 h}\right)=1+x^{3}(x+1) M^{2 h-1}$ is irreducible. Hence $M^{2 h}+\cdots+M=x^{3}(x+1) M^{2 h-1}$, so that $2 h-1=1$ and $M=1+x^{3}(x+1)$. It is impossible.

- If $U_{2 h}=T_{0, h}=x(x+1)^{3} M^{2 h-1}$, then $\sigma\left(M^{2 h}\right)$ is irreducible and equals $1+x(x+1)^{3} M^{2 h-1}$. Hence, as above, $h=1$ and $\sigma\left(M^{2 h}\right)=\left(x^{2}+x+1\right)\left(x^{6}+\right.$ $\left.x^{5}+x^{4}+x^{2}+1\right)$, which is not irreducible.

\section{Case $r=1$}

Lemma 5.1. For any $h \in \mathbb{N}^{*}, U_{2 h} \neq x^{6}(x+1)^{2} M^{2 h-2}=S_{1, h}$.

Proof. If $U_{2 h}=x^{6}(x+1)^{2} M^{2 h-2}$, then by Corollary 2.7, $\sigma\left(M^{2 h}\right)=$ $\left(1+x^{u}(x+1) M^{w}\right)\left(\left(1+x^{6-u}(x+1) M^{2 h-2-w}\right)\right.$, where $u, w \in \mathbb{N}, 1 \leqslant u \leqslant 5$. Hence

$$
M^{2 h}+\cdots+M+1=1+x^{u}(x+1) M^{w}+x^{6-u}(x+1) M^{2 h-2-w}+x^{6}(x+1)^{2} M^{2 h-2} .
$$


- If $w \neq 2 h-2-w$, then $\min (w, 2 h-2-w)=1$ and $M$ must divide $1+x^{c}(x+1)$, with $c \in\{u, 6-u\}$. This contradicts Lemma 5.3 below.

- If $w=2 h-2-w$ and $u=3$, then $M^{2 h}+\cdots+M+1=1+x^{6}(x+1)^{2} M^{2 h-2}$. Hence $2 h-2=1$. It is impossible.

- If $w=2 h-2-w$ and $u \neq 3$, then $w=h-1$,

$$
M^{2 h}+\cdots+M=(x+1) M^{h-1}\left(x^{u}+x^{6-u}\right)+x^{6}(x+1)^{2} M^{2 h-2} .
$$

So, $h-1=1, h=2$ and

$$
U_{4}=\sigma\left(\sigma\left(M^{4}\right)\right)=x^{2}(x+1)^{2}\left(x^{2}+x+1\right)\left(x^{10}+x^{9}+x^{8}+x^{6}+x^{4}+x^{3}+1\right) \neq S_{1,2} .
$$

Lemma 5.2. For any $h \in \mathbb{N}^{*}, U_{2 h} \neq x^{2}(x+1)^{6} M^{2 h-2}=T_{1, h}$.

Proof. If $U_{2 h}=x^{2}(x+1)^{6} M^{2 h-2}$, then as above, $\sigma\left(M^{2 h}\right)=\left(1+x(x+1)^{u} M^{w}\right)((1+$ $\left.x(x+1)^{6-u} M^{2 h-2-w}\right)$, where $u, w \in \mathbb{N}, 1 \leqslant u \leqslant 5$. Hence

$$
M^{2 h}+\cdots+M=x(x+1)^{u} M^{w}+x(x+1)^{6-u} M^{2 h-2-w}+x^{2}(x+1)^{6} M^{2 h-2} .
$$

- If $w \neq 2 h-2-w$, then $\delta:=\min (w, 2 h-2-w)=1$ and $M$ must divide $1+x(x+1)^{c}$, with $c \in\{u, 6-u\} \subset\{1, \ldots, 5\}$. Thus, $c=3=u=6-u$ and $M^{2 h-1}+\cdots+M+1=x(x+1)^{3}\left[M^{w-1}+M^{2 h-3-w}\right]+x^{2}(x+1)^{6} M^{2 h-3}$.

Remark that $M^{w-1}+M^{2 h-3-w}=1+M^{2 h-4}$ if $(w=\delta)$ or $(2 h-2-w=\delta)$. It follows that $M^{2 h-1}+\cdots+M^{2}=x(x+1)^{3} M^{2 h-4}+x^{2}(x+1)^{6} M^{2 h-3}$. So, $2 h-4=2, h=3$ and

$$
U_{6}=x^{5}(x+1)^{7}\left(1+x+x^{2}+x^{3}+x^{4}\right)^{2}\left(x^{4}+x^{3}+1\right) \neq x^{2}(x+1)^{6} M^{4}=T_{1,3} .
$$

- If $w=2 h-2-w$ and $u=3$, then $M^{2 h}+\cdots+M=x^{2}(x+1)^{6} M^{2 h-2}$. Hence $2 h-2=1$. It is impossible.

- If $w=2 h-2-w$ and $u \neq 3$, then $w=h-1$,

$$
M^{2 h}+\cdots+M=x M^{h-1}\left[(x+1)^{u}+(x+1)^{6-u}\right]+x^{2}(x+1)^{6} M^{2 h-2} .
$$

So, $h-1=1, h=2$ and

$$
U_{4}=\sigma\left(\sigma\left(M^{4}\right)\right)=x^{2}(x+1)^{2}\left(x^{2}+x+1\right)\left(x^{10}+x^{9}+x^{8}+x^{6}+x^{4}+x^{3}+1\right) \neq T_{1,2} .
$$

Lemma 5.3. For any $c \in \mathbb{N}, M$ does not divide $1+x^{c}(x+1)$.

Proof. Let $\alpha$ be a root of $M$. Then, one has $\alpha^{5}=1$ so that $\alpha^{c} \in\left\{1, \alpha, \ldots, \alpha^{4}\right\}$. Thus, $\alpha^{c}(\alpha+1) \neq 1$ for any $c \in \mathbb{N}$. We are done. 


\subsection{Case $r \geqslant 2$}

\section{Some precisions about divisors of $\sigma\left(M^{2 h}\right)$}

The polynomial $U$ defined below and its divisors will be useful:

$$
U:=\left(x^{2}+x+1\right)\left(x^{3}+x+1\right)\left(x^{3}+x^{2}+1\right)=x^{8}+x^{6}+x^{5}+x^{4}+x^{3}+x^{2}+1 .
$$

Moreover, it follows from Lemma 5.4 below, that we have to distinguish the following four cases:

i) $\operatorname{gcd}\left(\sigma\left(M^{2 h}\right), U\right)=1$,

ii) $\sigma\left(M^{2 h}\right)=\left(x^{2}+x+1\right) B$, with $\operatorname{gcd}(B, U)=1$,

iii) $\sigma\left(M^{2 h}\right)=\left(x^{3}+x+1\right)\left(x^{3}+x^{2}+1\right) B$, with $\operatorname{gcd}(B, U)=1$,

iv) $\sigma\left(M^{2 h}\right)=U B$, with $\operatorname{gcd}(B, U)=1$.

\section{Lemma 5.4.}

i) The polynomial $x^{3}+x+1$ divides $\sigma\left(M^{2 h}\right)$ if and only if $x^{3}+x^{2}+1$ divides $\sigma\left(M^{2 h}\right)$.

ii) If $x^{4}+x^{3}+1$ divides $\sigma\left(M^{2 h}\right)$ then $x^{4}+x+1$ must divide $\sigma\left(M^{2 h}\right)$. The converse is false.

iii) No irreducible polynomial of degree 4 divides $\sigma\left(M^{2 h}\right)$.

iv) No irreducible polynomial of degree 5 divides $\sigma\left(M^{2 h}\right)$.

Proof. i): Suppose that $x^{3}+x+1$ divides $\sigma\left(M^{2 h}\right)$ and let $\mu$ be a root of $x^{3}+x+1$. Then, one has $M(\mu)^{2 h+1}=1$. But, $M(\mu)=\mu^{4}+\mu^{2}=\mu^{2}(\mu+1)^{2}=\mu^{2} \mu^{6}=\mu^{8}=\mu$ because $\mu \in \mathbb{F}_{8}$. So, $\mu^{2 h+1}=1$ and 7 divides $2 h+1$.

Now, let $\beta$ be a root of $x^{3}+x^{2}+1$. Then, $M(\beta)=\beta^{4}+\beta=\beta^{3} \notin\{0,1\}$ because $\beta$ is of order 7 . Hence $M(\beta)^{7}=1$ so that $M(\beta)^{2 h+1}=1$ and $\left(x^{3}+x^{2}+1\right) \mid \sigma\left(M^{2 h}\right)$.

We similarly see that $\left(x^{3}+x+1\right) \mid \sigma\left(M^{2 h}\right)$ if $\left(x^{3}+x^{2}+1\right) \mid \sigma\left(M^{2 h}\right)$.

ii): Suppose that $x^{4}+x^{3}+1$ divides $\sigma\left(M^{2 h}\right)$ and let $\gamma$ be a root of $x^{4}+x^{3}+1$. Then, one has $M(\gamma)^{2 h+1}=1$. But, $M(\gamma)=\gamma(\gamma+1)=\frac{\gamma}{\gamma^{3}}$. So, $\left(\gamma^{-2}\right)^{2 h+1}=1$, $\gamma^{2 h+1}=1$. Since $\gamma^{15}=1, \gamma$ belonging to $\mathbb{F}_{16} \backslash\{0,1\}, \gamma^{3} \neq 1$ and $\gamma^{5} \neq 1$, we see that $\gamma$ is of order 15 . Thus, 15 divides $2 h+1$.

Now, let $\zeta$ be a root of $x^{4}+x+1$. Then, $M(\zeta)=\zeta^{3}+\zeta^{2}=\zeta^{2} \zeta^{4}=\zeta^{6} \notin\{0,1\}$. Hence $M(\zeta)^{15}=1$ so that $M(\zeta)^{2 h+1}=1$ and $\left(x^{4}+x+1\right) \mid \sigma\left(M^{2 h}\right)$. By taking $h=2$, we see that $\sigma\left(M^{2 h}\right)=\left(x^{4}+x+1\right)\left(x^{12}+x^{9}+x^{8}+x^{7}+x^{6}+x^{4}+x^{2}+x+1\right)$, so that the converse is not true.

iii) follows from ii) and from the fact that any irreducible divisor of $\sigma\left(M^{2 h}\right)$ must be of the form $1+x^{a_{i}}(x+1)^{b_{i}} M^{c_{i}}$ (Corollary 2.7), $x^{4}+x+1$ being not of this form.

iv) follows by an analogous proof, since any element of $\mathbb{F}_{32} \backslash\{0,1\}$ is of order 31 (a prime number), we see that if an irreducible polynomial of degree 5 divides $\sigma\left(M^{2 h}\right)$, then all irreducible polynomials of degree 5 divide it. But, $1+x+x^{2}+$ $x^{4}+x^{5}=1+x(x+1)^{2}\left(x^{2}+x+1\right)$ is irreducible of degree 5 and is not of the form $1+x^{a_{i}}(x+1)^{b_{i}} M^{c_{i}}$. This contradicts Corollary 2.7. 
$\alpha_{l}\left(M^{w}\right), \alpha_{l}\left(\sigma\left(M^{2 h}\right)\right)$ and $\alpha_{l}\left(U_{2 h}\right)$, for $l, w, h \in \mathbb{N}^{*}, l \leqslant 5$

In order to compute $\alpha_{l}\left(M^{w}\right), \alpha_{l}\left(\sigma\left(M^{2 h}\right)\right)$ and $\alpha_{l}\left(U_{2 h}\right)$, for $l, w, h \in \mathbb{N}^{*}$, we sometimes apply the following binomial coefficient properties obtained from the wellknown Lucas'Theorem (see [11]), without explicit mention. Some of our results are obtained by direct computations, so we omit their proofs.

Lemma 5.5. Let $n, k$ be two positive integers. Then, one has modulo 2:

i) $\left(\begin{array}{l}n \\ k\end{array}\right) \equiv 0$ if $n$ is even and $k$ odd.

ii) $\left(\begin{array}{l}n \\ k\end{array}\right) \equiv\left(\begin{array}{l}{\left[\frac{n}{2}\right]} \\ {\left[\frac{k}{2}\right]}\end{array}\right)$, otherwise.

iii) $\left(\begin{array}{c}2 n \\ n\end{array}\right) \equiv 0$.

Lemma 5.6. If $w \in \mathbb{N}^{*}$, then one has modulo 2 :

$$
\begin{aligned}
& \alpha_{1}\left(M^{w}\right)=w, \quad \alpha_{2}\left(M^{w}\right)=w+\left(\begin{array}{l}
w \\
2
\end{array}\right), \quad \alpha_{3}\left(M^{w}\right)=w+\left(\begin{array}{l}
w \\
3
\end{array}\right) \\
& \alpha_{4}\left(M^{w}\right)=\left(\begin{array}{l}
w \\
4
\end{array}\right)+w\left(\begin{array}{c}
w-1 \\
2
\end{array}\right)+w+\left(\begin{array}{c}
w \\
2
\end{array}\right), \\
& \alpha_{5}\left(M^{w}\right)=\left(\begin{array}{c}
w \\
5
\end{array}\right)+w\left(\begin{array}{c}
w-1 \\
3
\end{array}\right)+w\left(\begin{array}{c}
w-1 \\
2
\end{array}\right)+(w-2)\left(\begin{array}{l}
w \\
2
\end{array}\right) .
\end{aligned}
$$

In particular, for any $l \in \mathbb{N}^{*}$,

$$
\alpha_{1}\left(M^{2 l}\right)=\alpha_{3}\left(M^{2 l}\right)=0, \quad \alpha_{2}\left(M^{2 l}\right)=l, \quad \alpha_{4}\left(M^{2 l}\right)=\left(\begin{array}{l}
l \\
2
\end{array}\right)+l, \quad \alpha_{5}\left(M^{2 l}\right)=0 .
$$

Proof. Write

$$
M^{w}=\sum_{l=0}^{2}\left(\begin{array}{l}
w \\
l
\end{array}\right)\left(x^{4}+x^{3}\right)^{w-l}\left(x^{2}+x+1\right)^{l}+T, \quad \text { where } \operatorname{deg}\left(T_{1}\right) \leqslant 4 w-6,
$$

and consider all the coefficients of monomials of degree greater than $4 w-6$ in $\left(x^{4}+x^{3}\right)^{w}, w\left(x^{4}+x^{3}\right)^{w-1}\left(x^{2}+x+1\right)$ and in $\left(\begin{array}{c}w \\ l\end{array}\right)\left(x^{4}+x^{3}\right)^{w-2}\left(x^{2}+x+1\right)^{2}$.

Lemma 5.7. Let $u, v, w \in \mathbb{N}^{*}$ and $R_{v, w}=(x+1)^{v} M^{w}$. Then $\alpha_{k}\left(x^{u}(x+1)^{v} M^{w}\right)=$ $\alpha_{k}\left(R_{v, w}\right)$ and

$$
\begin{aligned}
& \alpha_{1}\left(R_{v, w}\right)=v+\alpha_{1}\left(M^{w}\right)=v+w, \quad \alpha_{2}\left(R_{v, w}\right)=\left(\begin{array}{l}
v \\
2
\end{array}\right)+v \alpha_{1}\left(M^{w}\right)+\alpha_{2}\left(M^{w}\right), \\
& \alpha_{3}\left(R_{v, w}\right)=\left(\begin{array}{l}
v \\
3
\end{array}\right)+\left(\begin{array}{l}
v \\
2
\end{array}\right) \alpha_{1}\left(M^{w}\right)+v \alpha_{2}\left(M^{w}\right)+\alpha_{3}\left(M^{w}\right), \\
& \alpha_{4}\left(R_{v, w}\right)=\left(\begin{array}{l}
v \\
4
\end{array}\right)+\left(\begin{array}{l}
v \\
3
\end{array}\right) \alpha_{1}\left(M^{w}\right)+\left(\begin{array}{l}
v \\
2
\end{array}\right) \alpha_{2}\left(M^{w}\right)+v \alpha_{3}\left(M^{w}\right)+\alpha_{4}\left(M^{w}\right), \\
& \alpha_{5}\left(R_{v, w}\right)=\left(\begin{array}{l}
v \\
5
\end{array}\right)+\left(\begin{array}{l}
v \\
4
\end{array}\right) w+\left(\begin{array}{l}
v \\
3
\end{array}\right) \alpha_{2}\left(M^{w}\right)+\left(\begin{array}{l}
v \\
2
\end{array}\right) \alpha_{3}\left(M^{w}\right)+v \alpha_{4}\left(M^{w}\right)+\alpha_{5}\left(M^{w}\right) .
\end{aligned}
$$


Proof. We easily see that $\alpha_{k}\left(x^{u}(x+1)^{v} M^{w}\right)=\alpha_{k}\left((x+1)^{v} M^{w}\right)$. We write $M^{w}=x^{4 w}+\sum_{l=1}^{5} \alpha_{l}\left(M^{w}\right) x^{4 w-l}+T_{2}$ and $(x+1)^{v}=\sum_{l=0}^{5}\left(\begin{array}{l}v \\ l\end{array}\right) x^{v-l}+T_{3}$, where $\operatorname{deg}\left(T_{2}\right) \leqslant 4 w-6$ and $\operatorname{deg}\left(T_{3}\right) \leqslant v-6$. As above, it suffices to consider the coefficients of all monomials of degree greater than $4 w-6$ in

$$
\left(x^{4 w}+\sum_{l=1}^{5} \alpha_{l}\left(M^{w}\right) x^{4 w-l}\right)\left(\sum_{l=0}^{5}\left(\begin{array}{l}
v \\
l
\end{array}\right) x^{v-l}\right) .
$$

From Lemma 5.7 and from the fact that $S_{r, h}$ and $T_{r, h}$ are squares, we get

Corollary 5.8. If $r, h \in \mathbb{N}^{*}$, with $r \geqslant 2$, then one has modulo 2 :

$$
\begin{gathered}
\alpha_{l}\left(S_{r, h}\right)=\alpha_{l}\left(T_{r, h}\right)=0 \quad \text { if } l \text { is odd } \\
\alpha_{2}\left(S_{r, h}\right)=\alpha_{2}\left(T_{r, h}\right)=h, \quad \alpha_{4}\left(S_{r, h}\right)=\alpha_{4}\left(T_{r, h}\right)=2^{r-2}+\left(\begin{array}{c}
h-2^{r-1} \\
2
\end{array}\right)+h .
\end{gathered}
$$

Lemma 5.9. For $h \in \mathbb{N}^{*}$, one has modulo $2: \alpha_{1}\left(\sigma\left(M^{2 h}\right)\right)=\alpha_{3}\left(\sigma\left(M^{2 h}\right)\right)=0$, $\alpha_{2}\left(\sigma\left(M^{2 h}\right)\right)=h, \alpha_{4}\left(\sigma\left(M^{2 h}\right)\right)=\left(\begin{array}{c}h-1 \\ 2\end{array}\right)$ and $\alpha_{5}\left(\sigma\left(M^{2 h}\right)\right)=1$.

Proof. Since $\sigma\left(M^{2 h}\right)=M^{2 h}+M^{2 h-1}+T$, with $\operatorname{deg}(T) \leqslant 4(2 h-2)=8 h-8$, one has $\alpha_{l}\left(\sigma\left(M^{2 h}\right)\right)=\alpha_{l}\left(M^{2 h}\right)$ if $1 \leqslant l \leqslant 3$, and $\alpha_{l}\left(\sigma\left(M^{2 h}\right)\right)=\alpha_{l}\left(M^{2 h}+M^{2 h-1}\right)=$ $\alpha_{l}\left(x(x+1)^{3} M^{2 h-1}\right)=\alpha_{l}\left(R_{3,2 h-1}\right)$ if $4 \leqslant l \leqslant 5$.

From Lemmas 5.7 and 5.6, one has modulo 2:

$$
\begin{aligned}
\alpha_{4}\left(R_{3,2 h-1}\right) & =\alpha_{1}\left(M^{2 h-1}\right)+\alpha_{2}\left(M^{2 h-1}\right)+\alpha_{3}\left(M^{2 h-1}\right)+\alpha_{4}\left(M^{2 h-1}\right) \\
& =\left(\begin{array}{c}
2 h-1 \\
3
\end{array}\right)+\left(\begin{array}{c}
2 h-1 \\
4
\end{array}\right)+\left(\begin{array}{c}
2 h-1 \\
2
\end{array}\right) \\
& =\left(\begin{array}{c}
h-1 \\
1
\end{array}\right)+\left(\begin{array}{c}
h-1 \\
2
\end{array}\right)+\left(\begin{array}{c}
h-1 \\
1
\end{array}\right) . \\
\alpha_{5}\left(R_{3,2 h-1}\right) & =\alpha_{2}\left(M^{2 h-1}\right)+\alpha_{3}\left(M^{2 h-1}\right)+\alpha_{4}\left(M^{2 h-1}\right)+\alpha_{5}\left(M^{2 h-1}\right) \\
& =1+\alpha_{4}\left(R_{3,2 h-1}\right)+\left(\begin{array}{c}
2 h-1 \\
5
\end{array}\right)+\left(\begin{array}{c}
2 h-2 \\
3
\end{array}\right)+\left(\begin{array}{c}
2 h-2 \\
2
\end{array}\right) \\
& =1+\left(\begin{array}{c}
h-1 \\
2
\end{array}\right)+\left(\begin{array}{c}
h-1 \\
2
\end{array}\right)+\left(\begin{array}{c}
h-1 \\
1
\end{array}\right)+\left(\begin{array}{c}
h-1 \\
1
\end{array}\right) .
\end{aligned}
$$

So, $\alpha_{4}\left(\sigma\left(M^{2 h}\right)\right)=\left(\begin{array}{c}h-1 \\ 2\end{array}\right)$ and $\alpha_{5}\left(\sigma\left(M^{2 h}\right)\right)=1$.

Lemma 5.10. Let $S \in \mathbb{F}_{2}[x]$ be such that no irreducible polynomial of degree at most 5 divides $S$. Then $\alpha_{l}(\sigma(S))=\alpha_{l}(S)$, for any $1 \leqslant l \leqslant 5$.

Proof. One has: $\sigma(S)=S+T$, where $\operatorname{deg}(T) \leqslant \operatorname{deg}(S)-6$. We are done.

Corollary 5.11. Let $h \in \mathbb{N}^{*}$ be such that $\operatorname{gcd}\left(\sigma\left(M^{2 h}\right), U\right)=1$. Then

$$
\alpha_{1}\left(U_{2 h}\right)=0, \quad \alpha_{2}\left(U_{2 h}\right)=h, \quad \alpha_{3}\left(U_{2 h}\right)=0, \quad \alpha_{4}\left(U_{2 h}\right)=\left(\begin{array}{c}
h-1 \\
2
\end{array}\right) .
$$


Proof. Apply Lemma 5.10 to $S=\sigma\left(M^{2 h}\right)$ by taking account of Corollary 2.7 and of Lemma 5.9.

Corollary 5.12. If $r, h \in \mathbb{N}^{*}$ with $\operatorname{gcd}\left(\sigma\left(M^{2 h}\right), U\right)=1$ and $r \geqslant 2$, then

$$
\alpha_{4}\left(U_{2 h}\right) \neq \alpha_{4}\left(S_{r, h}\right), \quad \alpha_{4}\left(U_{2 h}\right) \neq \alpha_{4}\left(T_{r, h}\right) .
$$

Lemma 5.13. Let $h \in \mathbb{N}^{*}$ be such that $\sigma\left(M^{2 h}\right)=\left(x^{2}+x+1\right) B$, where $\operatorname{gcd}(B, U)=1$. Then $\alpha_{1}\left(U_{2 h}\right)=0$ and $\alpha_{2}\left(U_{2 h}\right)=h+1$.

Proof. By Corollary 2.7, since $B$ divides $\sigma\left(M^{2 h}\right)$, we may apply Lemma 5.10 to $S=B$. One has, for any $1 \leqslant l \leqslant 5, \alpha_{l}(\sigma(B))=\alpha_{l}(B)$.

We may write: $B=x^{b}+\alpha_{1}(B) x^{b-1}+\alpha_{2}(B) x^{b-2}+\cdots$ and $\sigma\left(M^{2 h}\right)=$ $\left(x^{2}+x+1\right) B=x^{b+2}+\left(\alpha_{1}(B)+1\right) x^{b+1}+\left(\alpha_{2}(B)+1\right) x^{b}+\cdots$ So, $\alpha_{1}\left(\sigma\left(M^{2 h}\right)\right)=$ $\alpha_{1}(B)+1, \alpha_{2}\left(\sigma\left(M^{2 h}\right)\right)=\alpha_{2}(B)+\alpha_{1}(B)+1$.

On the other hand,

$U_{2 h}=\left(x^{2}+x\right) \sigma(B)=x^{b+2}+\left(\alpha_{1}(\sigma(B))+1\right) x^{b+1}+\left(\alpha_{2}(\sigma(B))+\alpha_{1}(\sigma(B))\right) x^{b}+\cdots$

Thus, $\alpha_{1}\left(U_{2 h}\right)=\alpha_{1}(\sigma(B))+1=\alpha_{1}(B)+1=\alpha_{1}\left(\sigma\left(M^{2 h}\right)\right)$ and

$$
\alpha_{2}\left(U_{2 h}\right)=\alpha_{2}(\sigma(B))+\alpha_{1}(\sigma(B))=\alpha_{2}(B)+\alpha_{1}(B)=\alpha_{2}\left(\sigma\left(M^{2 h}\right)\right)+1 .
$$

We get then our results from Lemma 5.9.

Corollary 5.14. If $r, h \in \mathbb{N}^{*}$ are such that $\sigma\left(M^{2 h}\right)=\left(x^{2}+x+1\right) B$, where $\operatorname{gcd}(B, U)=1$ and $r \geqslant 2$, then $\alpha_{2}\left(U_{2 h}\right)=h+1 \neq h=\alpha_{2}\left(S_{r, h}\right)=\alpha_{2}\left(T_{r, h}\right)$.

Lemma 5.15. Let $h \in \mathbb{N}^{*}$ be such that $\sigma\left(M^{2 h}\right)=\left(x^{3}+x+1\right)\left(x^{3}+x^{2}+1\right) B$, where $\operatorname{gcd}(B, U)=1$. Then

$$
\begin{gathered}
\alpha_{1}\left(U_{2 h}\right)=0, \quad \alpha_{2}\left(U_{2 h}\right)=h, \quad \alpha_{3}\left(U_{2 h}\right)=0, \\
\alpha_{4}\left(U_{2 h}\right)=1+\left(\begin{array}{c}
h-1 \\
2
\end{array}\right), \quad \alpha_{5}\left(U_{2 h}\right)=1 .
\end{gathered}
$$

Proof. We proceed as in the proof of Lemma 5.13. We give relations between the $\alpha_{l}\left(\sigma\left(M^{2 h}\right)\right)$ 's and the $\alpha_{l}\left(U_{2 h}\right)$ 's and apply Lemma 5.9.

By writing:

$$
\sigma\left(M^{2 h}\right)=\left(x^{6}+\cdots+x+1\right) B, \quad \text { with } B=x^{b}+\sum_{k=1}^{5} \alpha_{k}(B) x^{b-k}+\cdots,
$$

we get:

$$
\begin{aligned}
& \alpha_{1}\left(\sigma\left(M^{2 h}\right)\right)=\alpha_{1}(B)+1, \alpha_{2}\left(\sigma\left(M^{2 h}\right)\right)=\alpha_{2}(B)+\alpha_{1}(B)+1, \\
& \alpha_{3}\left(\sigma\left(M^{2 h}\right)\right)=\alpha_{3}(B)+\alpha_{2}(B)+\alpha_{1}(B)+1=\alpha_{3}(B)+\alpha_{2}\left(\sigma\left(M^{2 h}\right)\right), \\
& \alpha_{4}\left(\sigma\left(M^{2 h}\right)\right)=\alpha_{4}(B)+\alpha_{3}\left(\sigma\left(M^{2 h}\right)\right), \\
& \alpha_{5}\left(\sigma\left(M^{2 h}\right)\right)=\sum_{k=1}^{5} \alpha_{k}(B)+1=\sum_{k=2}^{5} \alpha_{k}(B)+\alpha_{1}\left(\sigma\left(M^{2 h}\right)\right) .
\end{aligned}
$$


Since $U_{2 h}=\left(x^{3}+x\right)\left(x^{3}+x^{2}\right) \sigma(B)=\left(x^{6}+x^{5}+x^{4}+x^{3}\right) \sigma(B)$, we obtain

$$
\begin{aligned}
& \alpha_{1}\left(U_{2 h}\right)=\alpha_{1}(\sigma(B))+1=\alpha_{1}(B)+1=\alpha_{1}\left(\sigma\left(M^{2 h}\right)\right), \\
& \alpha_{2}\left(U_{2 h}\right)=\alpha_{2}(\sigma(B))+\alpha_{1}(\sigma(B))+1=\alpha_{2}\left(\sigma\left(M^{2 h}\right)\right), \\
& \alpha_{3}\left(U_{2 h}\right)=\alpha_{3}(\sigma(B))+\alpha_{2}(\sigma(B))+\alpha_{1}(\sigma(B))+1=\alpha_{3}\left(\sigma\left(M^{2 h}\right)\right), \\
& \alpha_{4}\left(U_{2 h}\right)=\sum_{k=1}^{4} \alpha_{k}(\sigma(B))=\alpha_{4}\left(\sigma\left(M^{2 h}\right)\right)+1, \\
& \alpha_{5}\left(U_{2 h}\right)=\sum_{k=2}^{5} \alpha_{k}(\sigma(B))=\sum_{k=2}^{5} \alpha_{k}(B)=\alpha_{5}\left(\sigma\left(M^{2 h}\right)\right)+\alpha_{1}\left(\sigma\left(M^{2 h}\right)\right) .
\end{aligned}
$$

Corollary 5.16. If $r, h \in \mathbb{N}^{*}$ are such that $\sigma\left(M^{2 h}\right)=\left(x^{3}+x+1\right)\left(x^{3}+x^{2}+1\right) B$, where $\operatorname{gcd}(B, U)=1$ and $r \geqslant 2$, then $\alpha_{5}\left(U_{2 h}\right)=1 \neq 0=\alpha_{5}\left(S_{r, h}\right)=\alpha_{5}\left(T_{r, h}\right)$.

Lemma 5.17. Let $h \in \mathbb{N}^{*}$ be such that $\sigma\left(M^{2 h}\right)=U \cdot B$, where $\operatorname{gcd}(B, U)=1$. Then $\alpha_{1}\left(U_{2 h}\right)=0, \alpha_{2}\left(U_{2 h}\right)=h+1$ and $\alpha_{3}\left(U_{2 h}\right)=1$.

Proof. As above, we write:

$$
\sigma\left(M^{2 h}\right)=U B=\left(x^{8}+x^{6}+\cdots+x^{2}+1\right) B
$$

with $B=x^{b}+\sum_{k=1}^{3} \alpha_{k}(B) x^{b-k}+\cdots$

We get: $\alpha_{1}\left(\sigma\left(M^{2 h}\right)\right)=\alpha_{1}(B), \alpha_{2}\left(\sigma\left(M^{2 h}\right)\right)=\alpha_{2}(B)+1$ and $\alpha_{3}\left(\sigma\left(M^{2 h}\right)\right)=\alpha_{3}(B)+\alpha_{1}(B)+1$.

Here, $U_{2 h}=\left(x^{2}+x\right)\left(x^{3}+x\right)\left(x^{3}+x^{2}\right) \sigma(B)=\left(x^{8}+x^{4}\right) \sigma(B)$. So, one has:

$$
\begin{aligned}
& \alpha_{1}\left(U_{2 h}\right)=\alpha_{1}(\sigma(B))=\alpha_{1}(B)=\alpha_{1}\left(\sigma\left(M^{2 h}\right)\right), \\
& \alpha_{2}\left(U_{2 h}\right)=\alpha_{2}(\sigma(B))=\alpha_{2}(B)=\alpha_{2}\left(\sigma\left(M^{2 h}\right)\right)+1, \\
& \alpha_{3}\left(U_{2 h}\right)=\alpha_{3}(\sigma(B))=\alpha_{3}(B)=\alpha_{3}\left(\sigma\left(M^{2 h}\right)\right)+\alpha_{1}\left(\sigma\left(M^{2 h}\right)\right)+1 .
\end{aligned}
$$

Corollary 5.18. If $r, h \in \mathbb{N}^{*}$ are such that $\sigma\left(M^{2 h}\right)=U \cdot B$, where $\operatorname{gcd}(B, U)=1$ and $r \geqslant 2$, then $\alpha_{3}\left(U_{2 h}\right)=1 \neq 0=\alpha_{3}\left(S_{r, h}\right)=\alpha_{3}\left(T_{r, h}\right)$.

\section{References}

[1] J.T.B. Beard Jr, Perfect polynomials revisited, Publ. Math. Debrecen 38/1-2 (1991), 5-12.

[2] J.T.B. Beard Jr, J.R. Oconnell Jr, K.I. West, Perfect polynomials over $G F(q)$, Rend. Accad. Lincei 62 (1977), 283-291.

[3] E.F. Canaday, The sum of the divisors of a polynomial, Duke Math. J. 8 (1941), 721-737.

[4] L.H. Gallardo, O. Rahavandrainy, Odd perfect polynomials over $\mathbb{F}_{2}$, J. Théor. Nombres Bordeaux 19 (2007), 165-174. 
[5] L.H. Gallardo, O. Rahavandrainy, There is no odd perfect polynomial over $\mathbb{F}_{2}$ with four prime factors, Port. Math. (N.S.) 66(2) (2009), 131-145.

[6] L.H. Gallardo, O. Rahavandrainy, Even perfect polynomials over $\mathbb{F}_{2}$ with four prime factors Intern. J. of Pure and Applied Math. 52(2) (2009), 301-314.

[7] L.H. Gallardo, O. Rahavandrainy, All perfect polynomials with up to four prime factors over $\mathbb{F}_{4}$ Math. Commun. 14(1) (2009), 47-65.

[8] L.H. Gallardo, O. Rahavandrainy, On splitting perfect polynomials over $\mathbb{F}_{p^{p}}$, Int. Electron. J. Algebra 9 (2011), 85-102.

[9] L.H. Gallardo, O. Rahavandrainy, On even (unitary) perfect polynomials over $\mathbb{F}_{2}$, Finite Fields Appl. 18 (2012), 920-932.

[10] R. Kim, W. Koepf, Parity of the number of irreducible factors for composite polynomials, Finite Fields Appl. 16 (2010), 137-143.

[11] E. Lucas, Théorie des Fonctions Numériques Simplement Périodiques, Am. J. Math. 1(3) (1878), 197-240.

Address: Luis H. Gallardo and Olivier Rahavandrainy: Department of Mathematics, University of Brest, 6, Avenue Le Gorgeu, C.S. 93837, 29238 Brest Cedex 3, France.

E-mail: luisgall@univ-brest.fr, rahavand@univ-brest.fr

Received: 6 May 2015; revised: 1 February 2016 